\title{
Cardiovascular Complications of Modern Multiple Myeloma Therapy: Analysis of FDA Adverse Event Reporting System
}

Zaki Al-Yafeai ${ }^{1}$, Anil Ananthaneni ${ }^{{ }^{*}}$, Mohamed Ghoweba ${ }^{2^{*}}$, Hamzah Abduljabar ${ }^{1}$, David Aziz ${ }^{1}$, Udhayvir Grewal ${ }^{1}$

1Department of Internal Medicine, LSUHSC-Shreveport, Shreveport, LA. 2Department of Internal Medicine, CHRISTUS Good Shepherd /Texas A\&M College of Medicine, Longview, TX.

*equal contribution

Corresponding Author:

Zaki Al-Yafeai, MD, PhD

Internal Medicine Department

LSUHSC-Shreveport, Shreveport, LA

Key words:

Multiple myeloma, FAERS, elotuzumab, Ixazomib, daratumumab, and panobinostat. 


\section{Abstract:}

Purpose: Multiple myeloma accounts for over $15 \%$ of hematological malignancies. In attempt to tackle this malady, the FDA approved four drugs in 2015 which has propagated further development of new anti-multiple myeloma since. However, the health safety of these new agents is still ill-defined. The aim of this study is to delineate the cardiovascular adverse events of these drugs.

Methods: We searched the cardiac adverse events of the newly approved FDA drugs since 2015 using the U.S. Food and Drug Administration Adverse Events Reporting System database (FAERS). We calculated the reporting odds ratio (ROR) with 95\% confidence for four drugs that have the highest incidence of cardiovascular adverse events.

Results: Among the medications that have approved for MM Between 2015-2020, four novel drugs showed the highest incidence of cardiotoxicity. ROR $(95 \% \mathrm{Cl})$ for atrial fibrillation due to elotuzumab, Ixazomib, daratumumab, and panobinostat compared to other FAERS drugs was 5.8 (4.4-7.7), 1.93 (1.5-2.3), 4.8 (4.2-5.6), and 5.7 (4.1-8.1), respectively. The ROR (95\% Cl) for cardiac failure was 8.2 (6.4-10.5), 4.7 (4.1-5.4), 5.8 (4.9-6.7), and 5.6 (3.8-8.1) and ROR (95\% $\mathrm{Cl}$ ) for coronary disease was 2.7 (1.9-3.9), 2.7 (2.3-3.2), 2,3 (1.9-2.8), and 4.6 (3.2-6.6) due to elotuzumab, Ixazomib, daratumumab, and panobinostat versus all other drugs in FAERS.

Conclusions: The newly approved multiple myeloma therapy (elotuzumab, Ixazomib, daratumumab, panobinostat) are significantly associated with cardiotoxicity. These results highlight the importance of considering the cardiac history of patients with multiple myeloma when utilizing these novel agents. 


\section{Introduction:}

Multiple myeloma is characterized by neoplastic proliferation of plasma cells that produce monoclonal immunoglobulins. Proliferation of cells in the bone marrow often cause osteolytic lesions, osteopenia and/or pathologic fractures, subsequent hypercalcemia stemming from bone destruction and anemia mainly due to bone marrow replacement (Oyajobi, 2007). There is also observed renal dysfunction due to accumulation and precipitation of light chains which form casts in distal renal tubules (Dimopoulos, Kastritis, Rosinol, Blade, \& Ludwig, 2008). It is estimated that 35000 new cases are diagnosed annually, accounting for nearly $2 \%$ of all new cancer diagnoses ${ }^{3}$. Age-adjusted death rates have not changed significantly over 2009-2018 and have remained at around 3.1 per 100,000 population(Ludwig, Novis Durie, Meckl, Hinke, \& Durie, 2020).

While the initial therapy before newer therapeutic advances has classically consisted of VRd (bortezomib + lenalinomide + dexamethasone) and/or stem cell transplant with bortezomib/lenalinomide maintenance(Kumar et al., 2017), we have had newer approvals for treatment of multiple myeloma which have received at least one previous regimen. Between 2015-2016, the FDA approved 4 new drugs - elotuzumab (activates NK cells)(Magen \& Muchtar, 2016), ixazomib (proteosome inhibitor)(Raedler, 2016), daratumumab (targeting CD38)(Sanchez, Wang, Siegel, \& Wang, 2016), panobinostat(histone deacetylase inhibitor)(Laubach, Moreau, San-Miguel, \& Richardson, 2015) and more recently belantamab and selinexor in 2020(Joseph, Tai, Anderson, \& Lonial, 2021; Offidani, Corvatta, More, \& Olivieri, 2021). Clinical Daratumumab is frequently used in first line regimens along with VRd as D-VRd(Kumar et al., 2017).

While the usage of these drugs has increased in the recent years, there is little published evidence about their cardiovascular adverse effect profile. One of the largest trials, the CASTOR trial that included 283 and 286 patients in the VRd and D-VRd arms respectively reported negligible cardiovascular side effects -2 patients with atrial fibrillation in both arms, 1 patient with acute coronary syndrome among others in the D-VRd arm that could not reach statistical significance given low sample size(Palumbo et al., 2016). Therefore, we sought to analyze data from FAERS to study the reported cardiovascular adverse events of the newly approved multiple myeloma. 
medRxiv preprint doi: https://doi.org/10.1101/2021.12.20.21268138; this version posted February 14, 2022. The copyright holder for this preprint (which was not certified by peer review) is the author/funder, who has granted medRxiv a license to display the preprint in perpetuity.

It is made available under a CC-BY 4.0 International license .

\section{Methods:}

This is a retrospective adverse event analysis of the U.S. Food and Drug Administration's Adverse Event Reporting System (FEARS) database. FAERS is an online publicly available post-marketing pharmacovigilance database that records millions of adverse events, medication error reports, and product quality complaints submitted by healthcare professionals, manufacturers, and consumers worldwide for approved drugs and biologic products. Reports are evaluated by clinical reviewers in the Center for Drug Evaluation and Research and the Center for Biologics Evaluation and Research. The database includes data regarding the suspected pharmaceutical agent, indication for its use, patient characteristics, as well as the reported adverse event including its date of occurrence, nature, and outcome, among others. Since the data is anonymized, approval by an ethics committee was not required for this analysis. We aimed to evaluate the cardiovascular adverse events associated with newly FDAapproved multiple-myeloma therapeutic agents. Queries for the specific adverse event terms were performed (Supplemental). Statistical testing to measure the association of these drugs with the specified cardiac events of interest was performed using disproportionality signal analysis. These were presented as reporting odds ratios (RORs) of reported cardiac adverse events concomitant with the use of a newly approved multiple myeloma agent compared to the same reported adverse events within the entire database of pharmaceuticals within FAERS. The ROR was considered significant when the lower limit of the $95 \% \mathrm{Cl}$ was $>1.0$.

\section{Results:}

The total number of adverse events (AEs) from all drugs in the FAERS database was over 20 million. Of those, the total number of AEs caused by the newly-approved therapeutic agents for multiple myeloma (including in combination with other medications) evaluated in this study was 30,797 . Ixazomib had the highest number of total reported AEs with 13,701, followed by daratumumab with 10,235, while belantamab mafodotin had the lowest with 351 AEs. Out of the 30,797 AEs reported, 1131 were cardiac AEs. These included heart failure, atrial fibrillation, and coronary heart disease. Heart failure was reported the most with 470 AEs, while atrial fibrillation came next with 346, and coronary heart disease with 315 . Ixazomib had the highest reported heart failure and coronary heart disease AEs with 189 and 145 AEs respectively. Daratumumab had the highest atrial fibrillation AEs at 164 [Table 2]. 
Table 1 and supplemental tables 1-6 further demonstrate the characteristics of the cardiac related AEs associated with Elotuzumab, Ixazomib, Daratumumab, Panobinostat, Selinexor, and Belantamab mafodotin. As shown in these tables, male predominance was seen amongst all the drug-related AEs reported. For all the drugs, at least $98 \%$ of the reported AEs were classified as serious, with the mortality ranging from $19 \%-46 \%$ amongst these serious outcomes.

According to disproportionality signal analysis and compared to other medications, atrial fibrillation and coronary heart disease were mostly associated with the use of panobinostat with ROR of 5.7 (95\% Cl: 4.1-8.1, $\mathrm{P}<0.0001)$ and $4.6(95 \% \mathrm{Cl}: 3.8-8.1, \mathrm{P}<0.0001)$ and elotuzumab with ROR of 5.8 (95\% Cl: 4.4-7.7, $\mathrm{P}<0.0001)$ and 2.7 (95\% Cl: 1.9-3.9, $\mathrm{P}<0.0001)$ respectively. Coronary artery disease had the lowest ROR with all agents with odds ratio between 2.3-4.6. Heart failure was reported the most with the use of elotuzumab with ROR of 8.2 (95\% Cl: 6.410.5, $\mathrm{P}<0.0001)$, while ixazomib exhibited the least association with ROR of 4.7 (95\% Cl: 4.15.4, $\mathrm{P}<0.0001$ ). Of note, panobinostat's and daratumumab's ROR values were closely related compared to other agents [Figure $1 \mathrm{~A}-\mathrm{C}$ and Table 3].

\section{Discussion:}

The results of this study demonstrated an association between the use of the newest novel multiple myeloma agents and cardiotoxicity. Among all of the adverse events reported for these new agents on FAERS, cardiac complications represented little less than $10 \%$. Of all of the newly FDA approved multiple myeloma agents, elotuzumab, Ixazomib, daratumumab, panobinostat showed significant cardiac adverse events. Interestingly, cardiac failure, atrial fibrillation and coronary artery diseases were profoundly the highest of all reported cardiotoxicity. Interestingly Isatuximab, and belantamab mafodotin showed safer cardiac profile compared to elotuzumab, Ixazomib, daratumumab, panobinostat.

The fascinating Eloquent trials showed that elotuzumab significantly reduced multiple myeloma progression. Unspecified cardiac adverse events were reported among 7 patients with one death from heart failure with no concerning effects on QT prolongation(Dimopoulos et al., 2018; Passey, Darbenzio, Jou, Lynch, \& Gupta, 2016). The TOURMALINE trials that demonstrated the efficacy of Ixazomib on multiple myeloma reported similar adverse cardiac events (heart 
failure, myocardial infarction and arrhythmia) between the Ixazomib and the placebo group(Dimopoulos et al., 2020; Facon et al., 2021; Moreau et al., 2016). Three cases of myocardial infarction reported with panobinostat compared to placebo group(San-Miguel et al., 2014). Additionally, $17.6 \%$ of patient who received panobinostat and bortezomib and dexamethasone reported cardiac complications (most frequently atrial fibrillation, tachycardia, palpitation, and sinus tachycardia) on phase 3 PANORAMA-1 trial(Plummer, Driessen, Szabo, \& Mateos, 2019). Interestingly, no major cardiac adverse events reported associated with daratumumab(Dimopoulos et al., 2021; Palumbo et al., 2016).

While the findings of this study are novel, certain limitations pertains to this study. The wholistic profile of patient (comorbidities, risk factors, family history...etc.) are unknown. Moreover, prior cardiovascular functioning and other cardiac disorders are not considered when performing these studies. Additionally, the fundamental mechanisms mediating the cardiotoxicity associated with the novel multiple myeloma agents remain elusive.

The significance of our data stems from the increasing use of the new agents for multiple myeloma. As addressed above, multiple clinical trials showed sporadic cardiac adversaries of the novel multiple myeloma agents, however, a more thorough investigation of the cardiovascular profile of these medications is lacking. Our study strongly agrees with the aforementioned studies and showed enhanced cardiotoxicity especially atrial fibrillation, coronary artery disease and heart failure. Based on our results, we recommend physicians to discuss the cardiovascular risk with patients upon starting these medications. Electrocardiography and echocardiogram maybe help establish baseline cardiac function prior initiating these regimens.

In conclusions, the novel multiple myeloma agents are associated with enhance atrial fibrillation, coronary artery disease and heart failure. Patient risk stratification and baseline cardiac functioning testing (electrocardiography and echocardiogram) should be considered upon initiating these agents.

\section{Acknowledgment}

None. 


\section{References:}

Dimopoulos, M. A., Dytfeld, D., Grosicki, S., Moreau, P., Takezako, N., Hori, M., . . San-Miguel, J. (2018). Elotuzumab plus Pomalidomide and Dexamethasone for Multiple Myeloma. N Engl J Med, 379(19), 1811-1822. doi: 10.1056/NEJMoa1805762

Dimopoulos, M. A., Kastritis, E., Rosinol, L., Blade, J., \& Ludwig, H. (2008). Pathogenesis and treatment of renal failure in multiple myeloma. Leukemia, 22(8), 1485-1493. doi: 10.1038/leu.2008.131

Dimopoulos, M. A., Špička, I., Quach, H., Oriol, A., Hájek, R., Garg, M., . . Lonial, S. (2020). Ixazomib as Postinduction Maintenance for Patients With Newly Diagnosed Multiple Myeloma Not Undergoing Autologous Stem Cell Transplantation: The Phase III TOURMALINE-MM4 Trial. J Clin Oncol, 38(34), 4030-4041. doi: 10.1200/jco.20.02060

Dimopoulos, M. A., Terpos, E., Boccadoro, M., Delimpasi, S., Beksac, M., Katodritou, E., ... Sonneveld, P. (2021). Daratumumab plus pomalidomide and dexamethasone versus pomalidomide and dexamethasone alone in previously treated multiple myeloma (APOLLO): an open-label, randomised, phase 3 trial. Lancet Oncol, 22(6), 801-812. doi: 10.1016/s1470-2045(21)00128-5

Facon, T., Venner, C. P., Bahlis, N. J., Offner, F., White, D. J., Karlin, L., \& Benboubker, L. (2021). Oral ixazomib, lenalidomide, and dexamethasone for transplant-ineligible patients with newly diagnosed multiple myeloma. 137(26), 3616-3628. doi: 10.1182/blood.2020008787

Joseph, N. S., Tai, Y. T., Anderson, K. C., \& Lonial, S. (2021). Novel Approaches to Treating Relapsed and Refractory Multiple Myeloma with a Focus on Recent Approvals of Belantamab Mafodotin and Selinexor. Clin Pharmacol, 13, 169-180. doi: 10.2147/CPAA.S288840

Kumar, S. K., Rajkumar, V., Kyle, R. A., van Duin, M., Sonneveld, P., Mateos, M. V., . . Anderson, K. C. (2017). Multiple myeloma. Nat Rev Dis Primers, 3, 17046. doi: 10.1038/nrdp.2017.46

Laubach, J. P., Moreau, P., San-Miguel, J. F., \& Richardson, P. G. (2015). Panobinostat for the Treatment of Multiple Myeloma. Clin Cancer Res, 21(21), 4767-4773. doi: 10.1158/1078-0432.CCR-15-0530

Ludwig, H., Novis Durie, S., Meckl, A., Hinke, A., \& Durie, B. (2020). Multiple Myeloma Incidence and Mortality Around the Globe; Interrelations Between Health Access and Quality, Economic Resources, and Patient Empowerment. Oncologist, 25(9), e1406-e1413. doi: 10.1634/theoncologist.2020-0141

Magen, H., \& Muchtar, E. (2016). Elotuzumab: the first approved monoclonal antibody for multiple myeloma treatment. Ther Adv Hematol, 7(4), 187-195. doi: 10.1177/2040620716652862

Moreau, P., Masszi, T., Grzasko, N., Bahlis, N. J., Hansson, M., Pour, L., . . Richardson, P. G. (2016). Oral Ixazomib, Lenalidomide, and Dexamethasone for Multiple Myeloma. N Engl J Med, 374(17), 1621-1634. doi: 10.1056/NEJMoa1516282

Offidani, M., Corvatta, L., More, S., \& Olivieri, A. (2021). Belantamab Mafodotin for the Treatment of Multiple Myeloma: An Overview of the Clinical Efficacy and Safety. Drug Des Devel Ther, 15, 2401-2415. doi: 10.2147/DDDT.S267404

Oyajobi, B. O. (2007). Multiple myeloma/hypercalcemia. Arthritis Res Ther, 9 Suppl 1, S4. doi: 10.1186/ar2168

Palumbo, A., Chanan-Khan, A., Weisel, K., Nooka, A. K., Masszi, T., Beksac, M., . . . Investigators, C. (2016). Daratumumab, Bortezomib, and Dexamethasone for Multiple Myeloma. N Engl J Med, 375(8), 754-766. doi: 10.1056/NEJMoa1606038

Passey, C., Darbenzio, R., Jou, Y. M., Lynch, M., \& Gupta, M. (2016). Effects of elotuzumab on QT interval and cardiac safety in patients with multiple myeloma. Cancer Chemother Pharmacol, 78(6), 1237-1244. doi: 10.1007/s00280-016-3182-8

Plummer, C., Driessen, C., Szabo, Z., \& Mateos, M. V. (2019). Management of cardiovascular risk in patients with multiple myeloma. Blood Cancer J, 9(3), 26. doi: 10.1038/s41408-019-0183-y 
medRxiv preprint doi: https://doi.org/10.1101/2021.12.20.21268138; this version posted February 14, 2022. The copyright holder for this preprint

(which was not certified by peer review) is the author/funder, who has granted medRxiv a license to display the preprint in perpetuity.

It is made available under a CC-BY 4.0 International license .

Raedler, L. A. (2016). Ninlaro (Ixazomib): First Oral Proteasome Inhibitor Approved for the Treatment of Patients with Relapsed or Refractory Multiple Myeloma. Am Health Drug Benefits, 9(Spec Feature), 102-105.

San-Miguel, J. F., Hungria, V. T., Yoon, S. S., Beksac, M., Dimopoulos, M. A., Elghandour, A., . . . Richardson, P. G. (2014). Panobinostat plus bortezomib and dexamethasone versus placebo plus bortezomib and dexamethasone in patients with relapsed or relapsed and refractory multiple myeloma: a multicentre, randomised, double-blind phase 3 trial. Lancet Oncol, 15(11), 11951206. doi: 10.1016/s1470-2045(14)70440-1

Sanchez, L., Wang, Y., Siegel, D. S., \& Wang, M. L. (2016). Daratumumab: a first-in-class CD38 monoclonal antibody for the treatment of multiple myeloma. $J$ Hematol Oncol, 9(1), 51. doi: 10.1186/s13045-016-0283-0

Statements and Declarations:

\section{Funding:}

The authors declare that this work was not supported by any funds or grants.

\section{Competing Interests:}

The authors have no relevant financial or non-financial interests to disclose

\section{Author Contributions}

All the authors contributed to the study design, writing and analysis. Data collection and analysis [Zaki Al-Yafeai, Anil Ananthaneni, Mohamed Ghoweba], design [ Zaki Al-Yafeai, Hamzah Abduljabar, David Aziz, and Udhayvir Grewal]. The first draft was written by [Zaki Al-Yafeai] with edits and revision from all the authors. All authors read and approved the manuscript.

\section{Data Availability}

The datasets from the current study are available from the corresponding author upon request 


\section{Table 1}

\begin{tabular}{|c|c|c|c|}
\hline & Atrial Fibrillation & Cardiac Failure & Coronary Artery Disease \\
\hline \multicolumn{4}{|l|}{ Elotuzumab } \\
\hline Total number of reported AEs & 50 & 61 & 26 \\
\hline \multicolumn{4}{|l|}{ Age in years } \\
\hline $18-64$ & 14 & 14 & 7 \\
\hline $65-85$ & 30 & 39 & 13 \\
\hline $85+$ & 0 & 1 & 0 \\
\hline Not Specified & 6 & 7 & 6 \\
\hline \multicolumn{4}{|l|}{ Outcome reported per AE } \\
\hline Hospitalization & 39 & 40 & 14 \\
\hline Life-threatening & 6 & 4 & 7 \\
\hline Death & 11 & 13 & 16 \\
\hline Other Outcome & 40 & 52 & 16 \\
\hline Disabled & 1 & 4 & 0 \\
\hline \multicolumn{4}{|l|}{ Ixazomib } \\
\hline Total number of reported AEs & 93 & 212 & 139 \\
\hline \multicolumn{4}{|l|}{ Age in years } \\
\hline $18-64$ & 10 & 21 & 21 \\
\hline $65-85$ & 61 & 125 & 77 \\
\hline $85+$ & 3 & 16 & 5 \\
\hline Not Specified & 19 & 50 & 36 \\
\hline \multicolumn{4}{|l|}{ Outcome reported per AE } \\
\hline Hospitalization & 72 & 145 & 88 \\
\hline Life-threatening & 5 & 14 & 34 \\
\hline Nonserious & 1 & 2 & 0 \\
\hline Death & 18 & 50 & 46 \\
\hline Other Outcome & 48 & 179 & 112 \\
\hline Disabled & 0 & 4 & 1 \\
\hline \multicolumn{4}{|l|}{ Daratumumab } \\
\hline Total number of reported AEs & 191 & 207 & 94 \\
\hline \multicolumn{4}{|l|}{ Age in years } \\
\hline $18-64$ & 24 & 31 & 19 \\
\hline $65-85$ & 115 & 99 & 52 \\
\hline $85+$ & 2 & 10 & 3 \\
\hline Not Specified & 50 & 67 & 20 \\
\hline \multicolumn{4}{|l|}{ Outcome reported per AE } \\
\hline Hospitalization & 116 & 134 & 70 \\
\hline Life-threatening & 12 & 35 & 24 \\
\hline Death & 36 & 69 & 29 \\
\hline Other Outcome & 134 & 139 & 37 \\
\hline Disabled & 1 & 4 & 4 \\
\hline \multicolumn{4}{|l|}{ Panobinostat } \\
\hline Total number of reported AEs & 33 & 32 & 19 \\
\hline \multicolumn{4}{|l|}{ Age in years } \\
\hline $7-12$ & 0 & 1 & 0 \\
\hline $18-64$ & 3 & 5 & 7 \\
\hline $65-85$ & 16 & 14 & 3 \\
\hline $85+$ & 0 & 1 & 0 \\
\hline Not Specified & 14 & 11 & 9 \\
\hline \multicolumn{4}{|l|}{ Outcome reported per AE } \\
\hline Hospitalization & 4 & 3 & 4 \\
\hline Life-threatening & 1 & 1 & 1 \\
\hline Nonserious & 0 & 0 & 0 \\
\hline Death & 1 & 1 & 1 \\
\hline Other Outcome & 3 & 4 & 5 \\
\hline
\end{tabular}

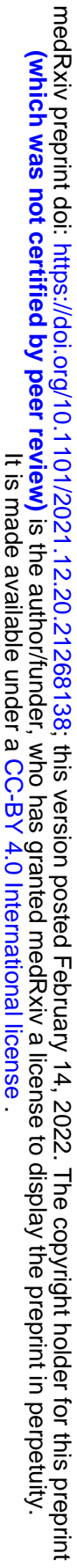




\section{able 2}

\begin{tabular}{|c|c|c|c|c|c|c|}
\hline Drug Name & $\begin{array}{c}\text { Total } \\
\text { (with } \\
\text { others+ } \\
\text { Alone) }\end{array}$ & $\begin{array}{l}\text { Heart } \\
\text { Failure }\end{array}$ & $\begin{array}{c}\text { Atrial } \\
\text { Flbrillation }\end{array}$ & $\begin{array}{l}\text { Coronary } \\
\text { Heart } \\
\text { Disease }\end{array}$ & $\begin{array}{c}\text { Total } \\
\text { Cardiac } \\
\text { Related AEs }\end{array}$ & $\begin{array}{c}\text { Total } \\
\text { Adverse } \\
\text { Events }\end{array}$ \\
\hline \multirow[t]{2}{*}{ Elotuzumab } & Total & $59(2.3 \%)$ & $49(2 \%)$ & $27(1 \%)$ & 135 & 2511 \\
\hline & Alone & 10 & 7 & 1 & 18 & \\
\hline \multirow[t]{2}{*}{ Ixazomib } & Total & $189(7.5 \%)$ & $89(3.5 \%)$ & $145(5.8 \%)$ & 423 & 13701 \\
\hline & Alone & 48 & 12 & 27 & 87 & \\
\hline \multirow[t]{2}{*}{ Daratumumab } & Total & $172(6.85 \%)$ & $164(6.5 \%)$ & $107(4.3 \%)$ & 443 & 10235 \\
\hline & Alone & 52 & 36 & 31 & 119 & \\
\hline \multirow[t]{2}{*}{ Panobinostat } & Total & $28(1.1 \%)$ & $33(1.3 \%)$ & $21(0.8 \%)$ & 82 & 1721 \\
\hline & Alone & 6 & 7 & 5 & 18 & \\
\hline \multirow{2}{*}{$\begin{array}{c}\text { Belantamab } \\
\text { mafodotin }\end{array}$} & Total & $3(0.1 \%)$ & $5(0.2 \%)$ & $6(0.2 \%)$ & 14 & 351 \\
\hline & Alone & 3 & 3 & 5 & 11 & \\
\hline \multirow[t]{2}{*}{ Selinexor } & Total & $19(0.76 \%)$ & $6(0.24 \%)$ & $9(0.36 \%)$ & 34 & 2278 \\
\hline & Alone & 8 & 3 & 6 & 17 & \\
\hline
\end{tabular}


A.

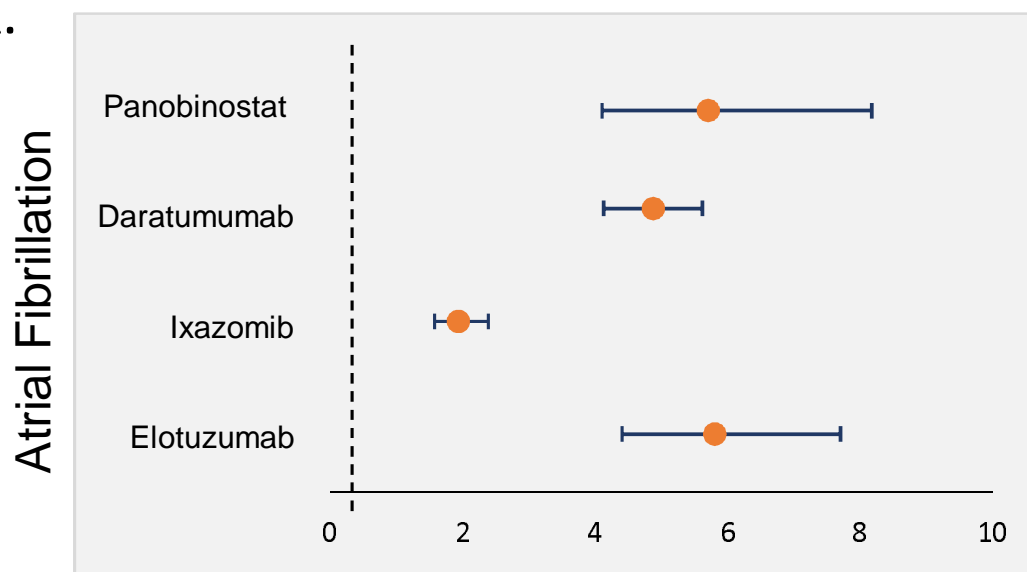

C.

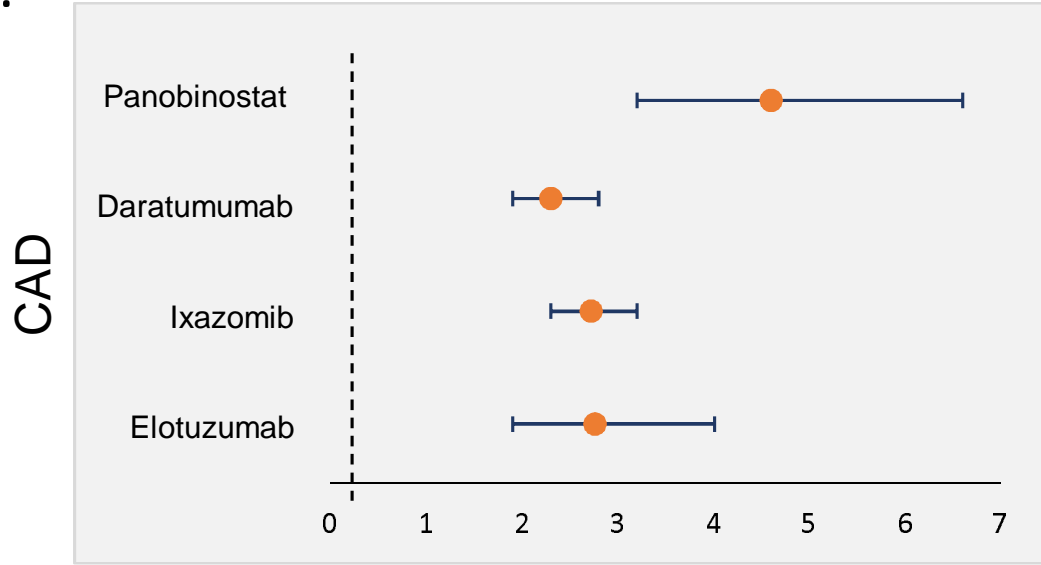

B.

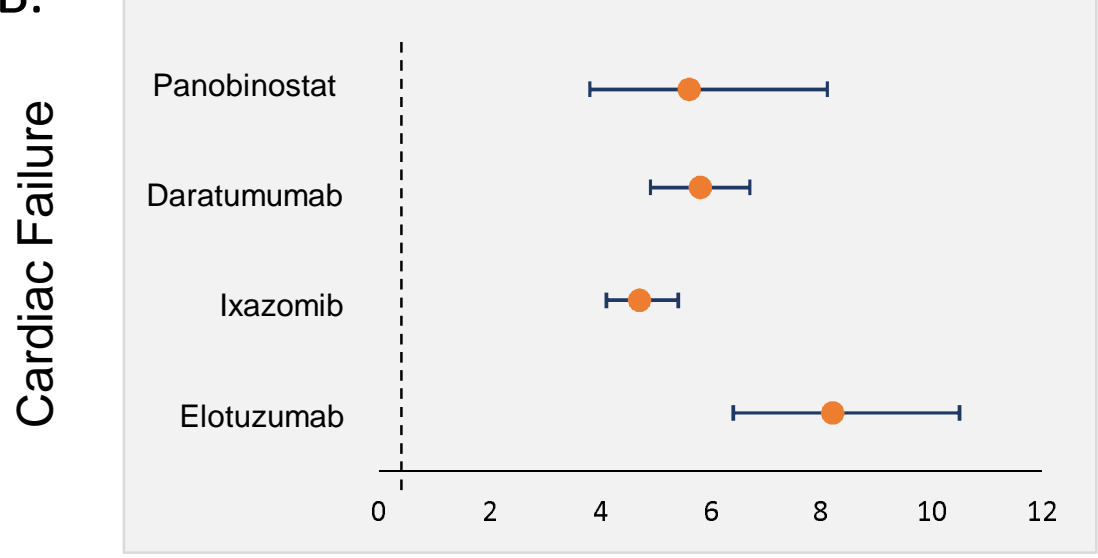

\section{Table 3}

\begin{tabular}{|c|c|c|c|c|}
\hline Drug name & $\begin{array}{l}\text { Elotuzumab vs } \\
\text { others }\end{array}$ & $\begin{array}{l}\text { lxazomib and } \\
\text { vs others }\end{array}$ & $\begin{array}{l}\text { Daratumumab vs } \\
\text { others }\end{array}$ & $\begin{array}{l}\text { Panobinostat vs } \\
\text { others }\end{array}$ \\
\hline $\begin{array}{l}\text { Atrial } \\
\text { Fibrillation }\end{array}$ & $\begin{array}{l}5.84(4.4-7.7) \\
P<0.0001\end{array}$ & $\begin{array}{l}1.93(1.55-2.36) \\
P<0.0001\end{array}$ & $\begin{array}{l}4.87(4.2-5.6) \\
P<0.0001\end{array}$ & $\begin{array}{l}5.74(4.1-8.1) \\
P<0.0001\end{array}$ \\
\hline Cardiac failure & $\begin{array}{l}8.2(6.4-10.5) \\
P:<0.0001\end{array}$ & $\begin{array}{l}4.7(4.1-5.4) \\
P:<0.0001\end{array}$ & $\begin{array}{l}5.8(4.9-6.7) \\
P<0.0001\end{array}$ & $\begin{array}{l}5.6(3.8-8.1) \\
P<0.0001\end{array}$ \\
\hline CAD & $\begin{array}{l}2.76(1.9-3.9) \\
P<0.0001\end{array}$ & $\begin{array}{l}2.7(2.3-3.2) \\
P<0.0001\end{array}$ & $\begin{array}{l}2.3(1.9-2.8) \\
P<0.0001\end{array}$ & $\begin{array}{l}4.6(3.2-6.6) \\
P<0.0001\end{array}$ \\
\hline
\end{tabular}

